\title{
Etanercept suppresses regenerative hyperplasia in psoriasis by acutely downregulating epidermal expression of interleukin (IL)-19, IL-20 and IL-24
}

F. Wang, ${ }^{1}$ N. Smith, ${ }^{1}$ L. Maier, ${ }^{1}$ W. Xia, ${ }^{1}$ C. Hammerberg, ${ }^{1}$ H. Chubb, ${ }^{1}$ C. Chen, ${ }^{1}$ M. Riblett, ${ }^{1}$ A. Johnston, ${ }^{1}$ J.E. Gudjonsson, ${ }^{1}$ Y. Helfrich, ${ }^{1}$ S. Kang, ${ }^{2}$ G.J. Fisher $^{1}$ and J.J. Voorhees ${ }^{1}$

${ }^{1}$ Department of Dermatology, University of Michigan, Ann Arbor, MI 48109, U.S.A.

${ }^{2}$ The Johns Hopkins Department of Dermatology, Baltimore, MD, U.S.A.

\section{Summary}

\section{Correspondence \\ Frank Wang. \\ E-mail: frawang@med.umich.edu \\ Accepted for publication \\ 17 March 2012 \\ Funding sources \\ Amgen Inc. provided partial funding and etanercept syringes for research purposes, but had no involvement in the design or conduct of the study or in the collection, management, analysis and interpretation of the data. Amgen was not involved in the preparation or review of the manuscript, or in publication decisions.}

\section{Conflicts of interest}

None declared.

DOI 10.1111/j.1365-2133.2012.10961.x
Background Psoriasis is a Th17/Th1-mediated skin disease that often responds to antitumour necrosis factor (TNF)- $\alpha$ therapies, such as etanercept.

Objectives To better define mechanisms by which etanercept improves psoriasis and to gain insight into disease pathogenesis.

Methods We investigated the early biochemical and cellular effects of etanercept on skin lesions in responder patients prior to substantial clinical improvement ( $\leq 4$ weeks).

Results By 1 week, etanercept acutely suppressed gene expression of the interleukin (IL)-20 subfamily of cytokines (IL-19, IL-20, IL-24), which were found to be predominantly epidermis-derived and which are implicated in stimulating epidermal hyperplasia. Additionally, by 1 week of therapy, suppression of other keratinocyte-derived products (chemokines, antimicrobial proteins) occurred, while suppression of epidermal regenerative hyperplasia occurred within 13 weeks. Th17 elements (IL-23p19, IL-12p40, IL-17A, IL-22) were suppressed by $3-4$ weeks. In vitro, TNF- $\alpha$ and IL-17A coordinately stimulated the expression of the IL-20 subfamily in normal keratinocytes.

Conclusions Based on the rapid suppression of regenerative hyperplasia, chemokines and other keratinocyte-derived products, including the IL-20 subfamily, we propose that epidermal activation is a very early target of etanercept. As many of these keratinocyte markers are stimulated by TNF- $\alpha$, their rapid downregulation is likely to reflect etanercept's antagonism of TNF- $\alpha$. Additionally, decreased epidermal hyperplasia might result specifically from acute suppression of the IL-20 subfamily, which is also a likely consequence of etanercept's antagonism of TNF- $\alpha$. Thus, the IL-20 subfamily has potential importance in the pathogenesis of psoriasis and therapeutic response to etanercept.
Psoriasis is characterized by epidermal hyperplasia and an infiltrate of dendritic cells (DCs), $\mathrm{T}$ cells, macrophages and neutrophils. Psoriasis is believed to be immune-mediated, with cytokines, chemokines, resident skin cells and infiltrating leucocytes creating an inflammatory network that culminates clinically in scaly, erythematous plaques, in which keratinocyte hyperproliferation and abnormal differentiation cause epidermal thickening. ${ }^{1-5}$

Importantly, DCs, T-helper 1 (Th1), T-helper 17 (Th17), and T-cytotoxic 17 and 22 (Tc17 and Tc22, respectively) cells produce cytokines involved in initiating and/or maintaining psoriatic plaques. ${ }^{1-4}$ Notable Th17-related cytokines include interleukin (IL)-23, IL-17A and IL-22. IL-23 is produced largely by 'inflammatory' DCs and stimulates development/maintenance of Th17 and, possibly, Tc17 cells. ${ }^{3,4,6}$ In turn, Th17 and Tc17 cells produce IL-17A and IL-22. Key producers of IL-22 in humans also include T-helper 22 and Th1 cells. ${ }^{7,8}$ IL-17A stimulates keratinocytes to produce antimicrobial peptides (AMPs) and chemokines, ${ }^{9-11}$ while IL-22 stimulates AMP production and epidermal hyperplasia. ${ }^{10,12-15}$ Additionally, mast cells and macrophages have been shown to produce IL-17A and IL-22, respectively, in psoriasis. ${ }^{16}$

In addition to Th17 cytokines, other potentially important cytokines include IL-19, IL-20 and IL-24, which are upregulated 
in psoriasis. ${ }^{17-19}$ Here, we define these cytokines as the 'IL-20 subfamily', ${ }^{15,20}$ as they share structural similarity, bind to the same receptors, activate signal transducer and activator of transcription (STAT) 3, and come from similar sources, including DCs, monocytes/macrophages and keratinocytes. ${ }^{17-25}$ The IL20 subfamily belongs to the IL-10 cytokine family, which also includes IL-22. Thus, IL-22 is closely related to the IL-20 subfamily, but is distinctly a Th17 cytokine (and therefore grouped separately here). Similar to IL-22, the IL-20 subfamily members stimulate epidermal hyperplasia in model systems. ${ }^{15}$ These cytokines also upregulate cytokeratin (CK) $16,{ }^{15,26}$ a marker of abnormal differentiation and/or regenerative hyperplasia that is overexpressed in psoriatic epidermis. ${ }^{27}$ Regenerative hyperplasia refers to keratinocyte hyperproliferation akin to that seen in wound healing. ${ }^{28}$

The importance of proinflammatory cytokines in the pathogenesis of psoriasis is substantiated by the efficacy of cytokine-based therapies, such as etanercept, adalimumab and infliximab, which target tumour necrosis factor (TNF)- $\alpha$. A recent study suggests that etanercept reduces epidermal thickening in psoriasis by targeting products of DCs and Th17 cells. $^{24,29}$ Here, we investigated the mechanisms of etanercept in vivo to gain additional insight into psoriasis pathogenesis. We examined early biochemical responses, prior to substantial clinical improvement. We found that etanercept rapidly suppresses many keratinocyte products, as well as epidermal expression of IL-19, IL-20 and IL-24, which is likely to reduce regenerative hyperplasia.

\section{Materials and methods}

\section{Patients}

The study (http://www.clinicaltrials.gov, NCT00111111) was approved by our Institutional Review Board. All patients provided written informed consent. Inclusion criteria included age $\geq 18$ years and stable plaque-type psoriasis involving $\geq 10 \%$ body surface area (BSA). Exclusion criteria included systemic therapy within 4 weeks, topical therapy within 2 weeks, or severe comorbidities. Subjects received etanercept $50 \mathrm{mg}$ twice weekly subcutaneously for 12 weeks. For clinical assessments, a single or two dermatologists evaluated the same subject throughout the study. For each subject, punch biopsies (6 mm) were obtained at baseline under local anaesthesia (lidocaine) from uninvolved skin and a target plaque. Subsequently, biopsies $(6 \mathrm{~mm})$ were obtained from the same plaque at weeks 1, 2, 3 and 4 for each subject. Biopsies were stored in OCT medium at $-80{ }^{\circ} \mathrm{C}$ for later analysis.

\section{Gene expression}

After RNA extraction (RNeasy Micro kit; Qiagen, Valencia, CA, U.S.A.), real-time reverse transcriptase-polymerase chain reaction (RT-PCR) was performed with primers/probes from Applied Biosystems (Carlsbad, CA, U.S.A.), as previously described. ${ }^{30}$ Expression was normalized to $36 \mathrm{~B} 4$.

\section{Laser capture microdissection}

Cryosections $(14 \mu \mathrm{m})$ were collected on to polyethylene naphthalate foil-coated slides (Leica Microsystems, Bannockburn, IL, U.S.A.) and stained with $0.5 \%$ thionin, according to manufacturer protocol (Leica LMD Protocol Guide). Using a Leica AS/LMD, epidermis and dermis were microdissected and collected separately in lysis buffer (RNeasy Micro kit; Qiagen) prior to RNA extraction and RT-PCR. Using an internal control, 36B4, expression levels were normalized to total obtained RNA, as described previously. ${ }^{31}$

\section{Immunostaining}

Cryosections $(7 \mu \mathrm{m})$ were stained, as previously described, ${ }^{30}$ using monoclonal antibodies against CK16 (Novocastra, Bannockburn, IL, U.S.A.), CK6 (Abcam, Cambridge, MA, U.S.A.), CK17 (Abcam), transglutaminase (Biochemical Technologies, Stoughton, MA, U.S.A.), Ki67 (Biogenex, San Ramon, CA, U.S.A.), CD11c (Dako, Carpinteria, CA, U.S.A.), CD3 (BD Pharmingen, Franklin Lakes, NJ, U.S.A.), CD4 (Biogenex) and CD8 (Biogenex). Area stained and epidermal thickness were quantified using Image-Pro Plus v4.1 (Media Cybernetics, Silver Spring, MD, U.S.A.). Isotype control for each primary antibody demonstrated no staining.

\section{In vitro experiments}

Normal human epidermal keratinocytes (NHEKs) were established as described previously, ${ }^{32}$ used in the second or third passage, grown to 4 days postconfluency, and starved of growth factors in unsupplemented M154 medium (Invitrogen/Cascade Biologics, Portland, OR, U.S.A.) for 24 h. Cultures were then stimulated with human IL-17A (10 $\left.\mathrm{ng} \mathrm{mL}{ }^{-1}\right)$ and/or TNF- $\alpha$ ( $1 \mathrm{ng} \mathrm{mL}^{-1}$ ) (both from R\&D Systems, Minneapolis, MN, U.S.A.) for $24 \mathrm{~h}$. Recombinant cytokines were dissolved in sterile filtered $0 \cdot 1 \%$ bovine serum albumin in phosphate-buffered saline (PBS). Control stimulations were done with vehicle only $(0 \cdot 1 \%$ bovine serum albumin in PBS). RNA was extracted as described above.

\section{Statistics}

Analyses are restricted to responders and are presented as means \pm SEM. Given the longitudinal nature of the design, the repeated measures model and maximum likelihood approach for estimating the parameters and their standard errors was used. In the mixed model, likelihood ratio tests were used to assess statistical significance, and time was coded with dummy variables. SAS v9.1 (SAS Institute Inc., Cary, NC, U.S.A.) was used to perform the analysis. The paired t-test was used to compare baseline lesional and uninvolved skin, and to analyse epidermal and dermal contributions in laser capture microdissection (LCM) experiments. One-way ANOvA with Tukey post-hoc comparisons were used to assess differences between contributions at each time point and to analyse 
in vitro data. When appropriate, logarithmic transformation was applied to achieve normality. All statistical tests were two sided with an overall alpha level of 0.05 used to determine significance.

\section{Results}

\section{Substantial clinical improvement by 3 months of therapy}

Patients received etanercept without severe adverse events. Using published criteria, ${ }^{33}$ we determined that 25 of 30 subjects were responders, defined as patients with Physicians' Global Assessment (PGA) $\leq 2$ at week 12 of therapy and improvement of PGA from baseline. PGA is a validated scale that correlates with Psoriasis Area and Severity Index. ${ }^{34}$ Mean \pm SEM PGA of responders at baseline was $3 \cdot 2 \pm 0 \cdot 1$, equivalent to 'moderate' disease, with improvement beginning at 2 weeks $(2.8 \pm 0.1, \quad P<0.001)$ and most substantial at 12 weeks $(1 \cdot 3 \pm 0 \cdot 1$, or 'minimal' disease, $P<0.001$, Fig. 1a). Individual clinical parameters, including erythema, induration and scaling, exhibited similar trends (Fig. 1b-d). At baseline, involved BSA in responders was $21 \pm 2 \%$, with improvement starting at 4 weeks $(18 \pm 2 \%, P=0.03)$ and most substantial at 12 weeks $(8 \pm 2 \%, \mathrm{P}<0.001$, Fig. $1 \mathrm{e})$. Overall, response rate and degree of improvement were consistent with earlier trials. ${ }^{24,33}$ Analyses of nonresponders $(n=5)$ were underpowered to detect differences due to small sample size, and therefore, only responder data are presented. Given limited tissue, the number of analysed responders ranged from seven to 20 for each marker.

\section{The interleukin-20 subfamily is acutely suppressed by etanercept and derived predominantly from psoriatic epidermis}

Consistent with previous reports, ${ }^{17-19,35}$ the IL-20 subfamily was overexpressed in baseline lesional skin, with gene expression of IL-19, IL-20, IL-24 and STAT3 increased 98-, 31-, 7·4- and 2-2-fold, respectively, compared with uninvolved skin (all $\mathrm{P}<0.001$, Table 1 ). By week 1 of therapy, IL-19, IL-20 and IL-24 expression decreased by 53\% ( $P=0.01)$, 57\% $(\mathrm{P}<0.001)$ and $66 \% \quad(\mathrm{P}=0.006)$, respectively, compared with baseline plaques (Fig. 2a). Additionally, expression of STAT3 decreased significantly by week $1(P=0 \cdot 02$, Fig. $2 a)$.

IL-19, IL-20 and IL-24 are derived from keratinocytes and leucocytes in the upper dermis. To determine the relative contribution of different cell types to production of these cytokines, we used laser capture microdissection (LCM) to separate untreated lesional epidermis and dermis. Gene expression of IL-19, IL-20, IL-24 and STAT3 was overwhelmingly greater in epidermis than dermis (all $\mathrm{P}<0.05$, Fig. $2 \mathrm{~b}$ ).

The IL-20 subfamily binds to receptors on keratinocytes, with IL-19, IL-20 and IL-24 binding to a complex consisting of IL-20 receptor 1 (IL-20R1) and IL-20 receptor 2 (IL20R2), and IL-20 and IL-24 binding to the complex IL-20R2/IL-22 receptor 1 (IL-22R1) (Fig. S1; see Supporting

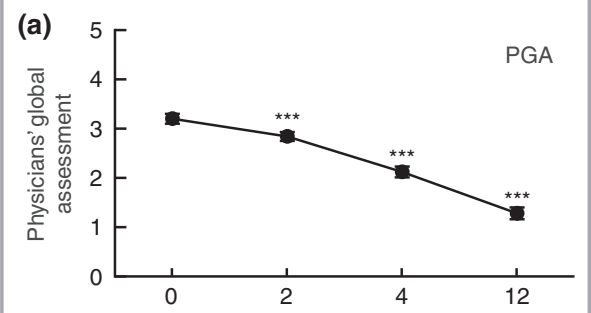

(b)

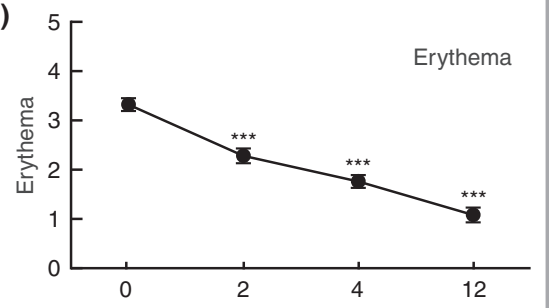

(c)

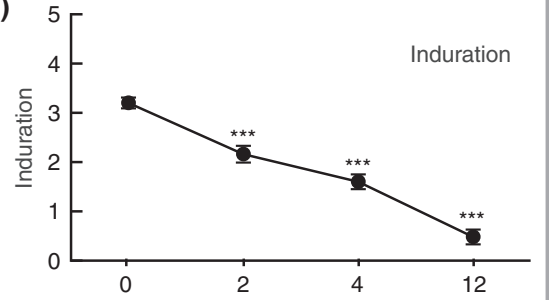

(d)
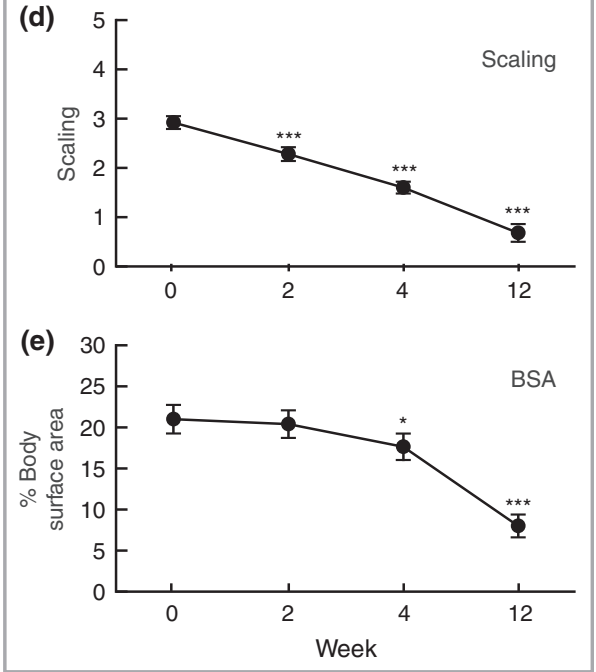

Fig 1. Substantial clinical improvement of psoriasis by 12 weeks of etanercept therapy. (a) Physicians' Global Assessment (PGA, $0=$ clear, 5 = severe), (b) erythema, (c) induration, (d) scaling and (e) involved body surface area (BSA) were determined in responder patients $(\mathrm{n}=25)$ at weeks 0 (baseline), 2, 4 and 12 of therapy. Data are presented as means \pm SEM. $* \mathrm{P}<0.05$, *** $<<0.001$, compared with baseline.

Information). ${ }^{19,25,36,37}$ Consistent with previous reports, ${ }^{18,35}$ gene expression of IL-20R1 and IL-20R2 demonstrated a significant decrease $(P=0.02)$ and downward trend, respectively, in untreated plaques, compared with uninvolved skin (Fig. S1; see Supporting Information). At week 3, expression of these subunits normalized $(\mathrm{P}<0.001)$. Expression of IL22R1 was increased in baseline plaques $(P=0.03)$, but did 
Table 1 Gene transcripts upregulated in psoriatic plaques at baseline

\begin{tabular}{|c|c|c|}
\hline mRNA transcript & Functional importance & $\begin{array}{l}\text { Fold change in } \\
\text { untreated lesional skin }\end{array}$ \\
\hline \multicolumn{3}{|l|}{ IL-20 subfamily } \\
\hline IL-19 & IL-20-related cytokine & 98 \\
\hline IL-20 & IL-20-related cytokine & 31 \\
\hline IL-24 & IL-20-related cytokine & $7 \cdot 4$ \\
\hline STAT3 & Downstream mediator of IL-20 subfamily & $2 \cdot 2$ \\
\hline \multicolumn{3}{|c|}{ Regenerative maturation markers } \\
\hline CK16 & $\begin{array}{l}\text { Upregulated in hyperproliferative } \\
\text { psoriatic keratinocytes }\end{array}$ & 22 \\
\hline CK17 & $\begin{array}{l}\text { Upregulated in hyperproliferative } \\
\text { psoriatic keratinocytes }\end{array}$ & $3 \cdot 4$ \\
\hline \multicolumn{3}{|c|}{ Markers of keratinocyte activation } \\
\hline CXCL1 & Neutrophil chemoattractant & 16 \\
\hline CXCL8 & Neutrophil chemoattractant & 68 \\
\hline CCL20 & T-cell and dendritic cell chemoattractant & 15 \\
\hline CXCL10 & T-cell chemoattractant & $3 \cdot 8$ \\
\hline IL- $1 \beta$ & Inflammatory cytokine & $6 \cdot 7$ \\
\hline hCAP-18/LL-37 & Antimicrobial peptide & $6 \cdot 8$ \\
\hline HBD-2 & Antimicrobial peptide & 1234 \\
\hline HBD-3 & Antimicrobial peptide & 100 \\
\hline AREG & Keratinocyte growth factor & $3 \cdot 6$ \\
\hline HB-EGF & Keratinocyte growth factor & $2 \cdot 4$ \\
\hline \multicolumn{3}{|c|}{ Markers of dendritic cell activation } \\
\hline IL-12p40 & Th17-related, inflammatory cytokine & $4 \cdot 9$ \\
\hline IL-23p19 & Th17-related, inflammatory cytokine & $2 \cdot 9$ \\
\hline iNOS & Inducible nitric oxide synthase & 11 \\
\hline \multicolumn{3}{|c|}{ Markers of T-cell activation } \\
\hline IL-17 A & Th17-related, inflammatory cytokine & 113 \\
\hline IL-22 & Th17-related, inflammatory cytokine & $6 \cdot 3$ \\
\hline \multicolumn{3}{|c|}{$\begin{array}{l}\text { IL, interleukin; STAT, signal transducer and activator of transcription; CK, cytokeratin; hCAP- } \\
\text { 18/LL-37, human cathelicidin antimicrobial peptide-18/leucine-leucine- } 37 \text {; HBD, human } \beta \text { - } \\
\text { defensin; AREG, amphiregulin; HB-EGF, heparin-binding epidermal growth factor-like growth } \\
\text { factor; iNOS, inducible nitric oxide synthase. }{ }^{a} \text { With respect to uninvolved skin. All fold } \\
\text { changes P }<0.001 \text {. }\end{array}$} \\
\hline
\end{tabular}

not normalize during 4 weeks of therapy (Fig. S1; see Supporting Information). Hence, etanercept therapy led to early suppression of IL-19, IL-20 and IL-24, which were predominantly derived from epidermis. This suppression was followed later by normalization of several of their receptor subunits.

\section{Rapid downregulation of regenerative hyperplasia}

Compared with uninvolved skin, baseline lesions exhibited elevated epidermal immunostaining of CK16 $(P<0.001$, Fig. 3a), which became significantly reduced by week 1 $(\mathrm{P}=0.001$, Fig. 3b). Interestingly, changes in CK16 protein expression preceded those of gene expression (Table 1), which exhibited a downward trend at weeks 2 and 3 and a significant decrease at week $4(\mathrm{P}=0.006$, Fig. 3c).

Expression of other hyperplasia markers also decreased. At week 1 , thickness of lesional epidermis decreased significantly ( $P=0.04$, Fig. $3 \mathrm{~d})$, and by week 2 , lesional epidermis demonstrated decreased Ki67 staining, indicating decreased keratinocyte proliferation ( $\mathrm{P}=0.006$, Fig. 3e). Likewise, CK17 immunostaining and gene expression decreased at week 2
$(P<0.001)$ and week $3(P=0.02)$, respectively (Fig. 3f, g) Finally, decreased immunostaining was observed at week 2 for CK6 $(P=0.007)$ and at week 4 for transglutaminase $(\mathrm{P}<0.001)$ (Fig. 3h, i). These data indicate that etanercept causes downregulation of the IL-20 subfamily concomitant with or prior to normalization of epidermal proliferation, differentiation and thickness.

\section{Suppression of keratinocyte activation}

Because regenerative hyperplasia was rapidly targeted by etanercept, we next examined changes in epidermal activation, as reflected by synthesis of proinflammatory products (Table 1 ). In lesional skin, gene expression of keratinocyte-derived chemokines, including CXCL1, CXCL8, CCL20 and CXCL10, was suppressed by 1 week (all $P<0 \cdot 01$, Fig. S2a-d; see Supporting Information).

Other proinflammatory products of keratinocytes also showed decreased gene expression at 1 week, including IL- $1 \beta$, as well as the AMPs human cathelicidin antimicrobial peptide18/eucine-leucine-37 (hCAP-18/LL-37) and human $\beta$-defensin 


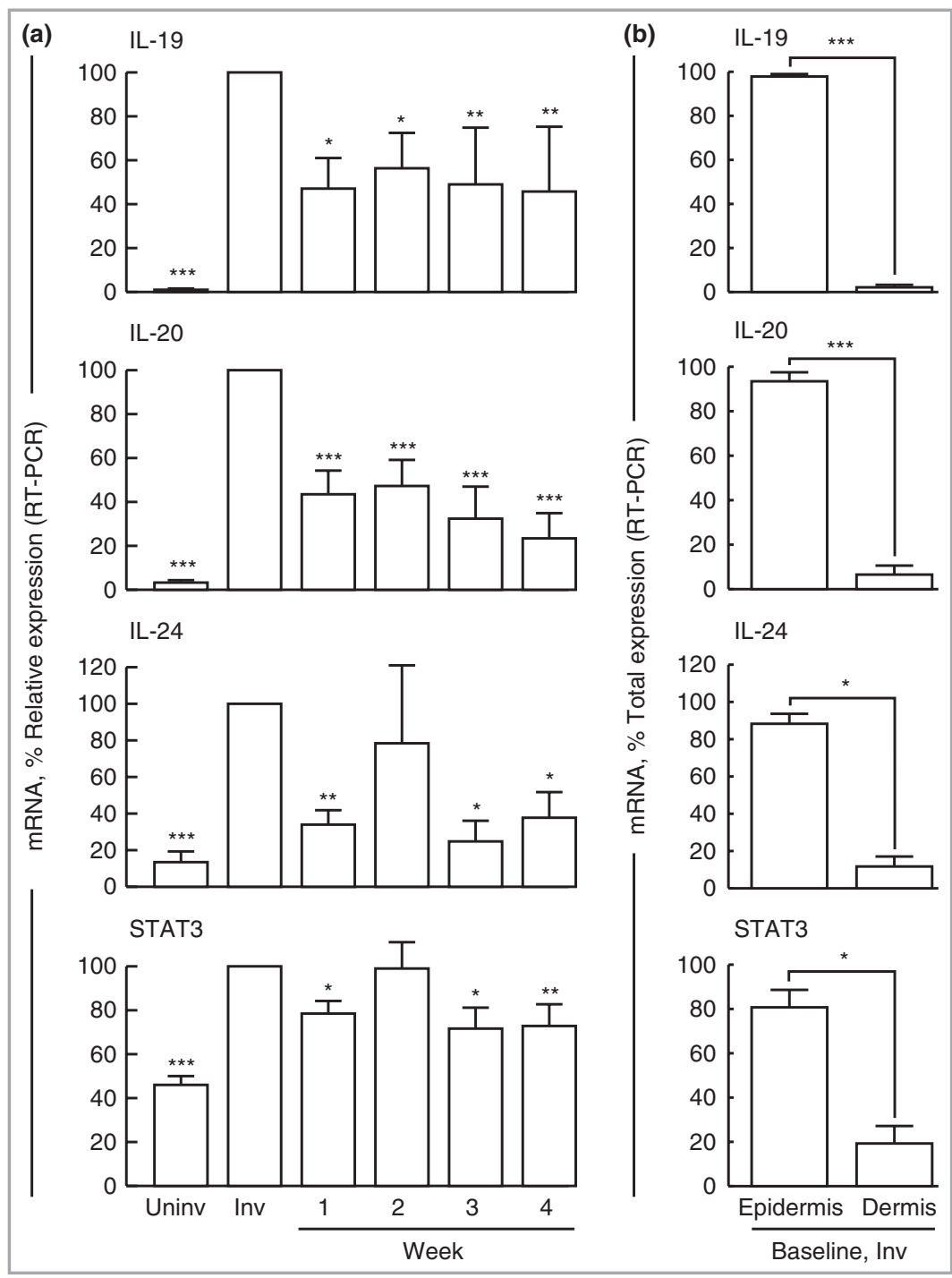

Fig 2. Interleukin (IL)-20-related cytokines are rapidly suppressed by 1 week of etanercept therapy and derived predominantly from epidermis. (a) In biopsies from uninvolved (Uninv) and involved (Inv) skin at baseline, as well as involved skin at the indicated weeks of therapy, real-time reverse transcriptase-polymerase chain reaction (RT-PCR) was used to determine expression of IL-19 ( $=17), \mathrm{IL}-20(\mathrm{n}=17), \mathrm{IL}-24$ $(\mathrm{n}=16)$ and signal transducer and activator of transcription (STAT) $3(\mathrm{n}=14)$. (b) To determine the relative contribution of different cell types to the production of IL-20-related cytokines, laser capture microdissection was used to separate epidermis and dermis in baseline involved skin, and expression of IL-19, IL-20, IL-24 and STAT3 (all $\mathrm{n}=6$ ) was determined using real-time RT-PCR. Data are derived from clinical responders, presented as percentage expression relative to baseline involved skin (normalized to $100 \%$ ), and shown as means \pm SEM. $* \mathrm{P}<0.05, * * \mathrm{P}<0.01$, $* * * \mathrm{p}<0.001$, compared with baseline involved skin (a), and epidermis vs. dermis (b).
(HBD)-2 and HBD-3 (all P $<0 \cdot 01$, Fig. S2e-h; see Supporting Information).

Lastly, in addition to the IL-20 subfamily, psoriatic keratinocytes produce growth factors that promote epidermal hyperplasia, including amphiregulin (AREG) and heparinbinding epidermal growth factor-like growth factor (HB-EGF) (Table 1). During therapy, gene expression of these growth factors decreased at week 1 and 2, respectively (both $P<0.05$, Fig. S2i, j; see Supporting Information). Altogether, the downregulation of epidermis-derived chemokines, cytokines, AMPs and growth factors indicates that etanercept readily suppresses activation of keratinocytes.

\section{Suppression of dendritic cell activation}

Inflammatory DCs produce IL-23, TNF- $\alpha$ and inducible nitric oxide synthase (iNOS).$^{3,4}$ In untreated plaques, gene expression of the IL-23 subunits p40 and p19 was significantly upregulated (both $\mathrm{P}<0.001$, Table 1). During therapy, expression of p40 and p19 did not decrease significantly until week $3 \quad(P=0.05)$ and week $4 \quad(P=0.02)$, respectively (Fig. 4a). Similarly, lesional expression of iNOS (Table 1) did not decrease until week 3 ( $P=0 \cdot 05$, Fig. 4a).

Inflammatory $\mathrm{DCs}$ are $\mathrm{CD} 11 \mathrm{c}+$ and localized primarily in lesional papillary dermis. ${ }^{3,5}$ At baseline, CD11c staining was increased $4 \cdot 1$-fold $(P=0 \cdot 005, n=7)$ in lesional dermis, compared with uninvolved dermis (Fig. S3; see Supporting Information). During therapy, dermal CD11c immunostaining did not show any trend towards decrease (Fig. S3; see Supporting Information), suggesting that substantial deletion/apoptosis of inflammatory DCs does not occur.

\section{Suppression of T-cell activation}

In psoriatic lesions, CD4+ T cells, including Th17 cells, are the predominant $\mathrm{T}$-cell population in the dermis, whereas CD8 + $\mathrm{T}$ cells predominate in the epidermis. ${ }^{16,38,39}$ Based on immunostaining of CD3, CD4 and CD8, T-cell numbers were significantly elevated in untreated plaques, compared with uninvolved skin (all $\mathrm{P}<0.05$, Fig. S4; see Supporting Information). 


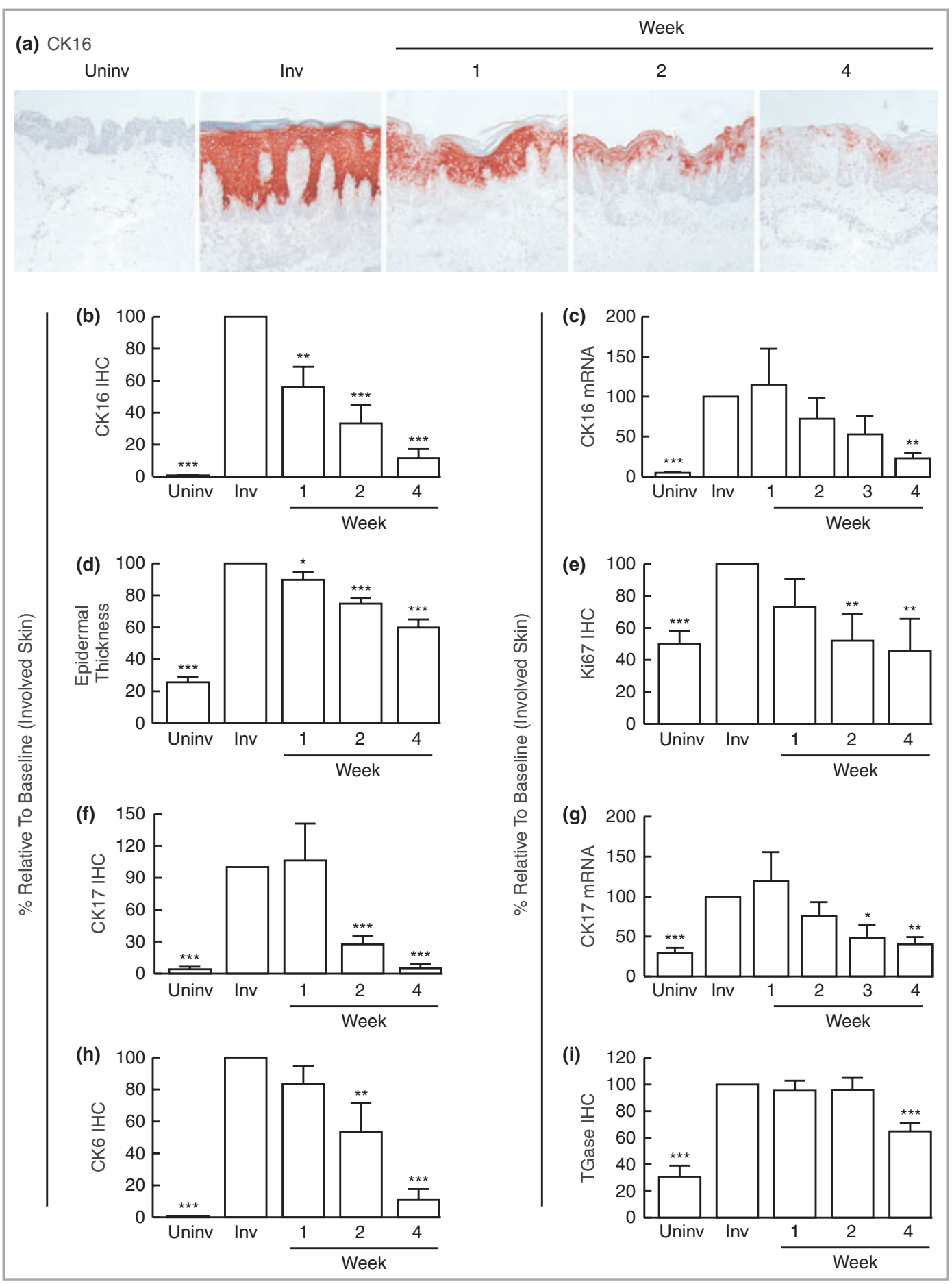

Fig 3. Suppression of epidermal hyperplasia by 1-4 weeks of etanercept therapy. In uninvolved (Uninv) and involved (Inv) skin at baseline, as well as involved skin at the indicated weeks of therapy, cytokeratin (CK) 16 was assessed by (a) immunostaining (original magnification $\times 10$, $\mathrm{n}=12$ ), (b) quantification of area stained $(\mathrm{n}=12)$ and $(\mathrm{c})$ gene expression $(\mathrm{n}=17)$. (d) Epidermal thickness $(\mathrm{n}=14)$ of histological sections was measured (baseline lesional epidermis $=307 \cdot 3 \pm 27 \cdot 2 \mu \mathrm{m}$ ) during therapy, while (e) keratinocyte hyperproliferation was assessed by epidermal immunostaining of Ki67 ( $\mathrm{n}=7)$. CK17 was assessed by $(\mathrm{f})$ area of epidermal immunostaining $(\mathrm{n}=8)$ and $(\mathrm{g})$ gene expression $(n=15)$. Lastly, area of epidermal immunostaining of other hyperplasia/differentiation markers, including (h) CK6 ( $=8)$ and (i) tranglutaminase (TGase, $n=8$ ), was also assessed. Data are derived from clinical responders, presented as percentage relative to baseline involved skin (normalized to $100 \%$ ), and shown as means \pm SEM. $* \mathrm{P}<0 \cdot 05, * * \mathrm{P}<0 \cdot 01, * * * \mathrm{P}<0 \cdot 001$, compared with baseline involved skin. IHC, immunohistochemistry. 


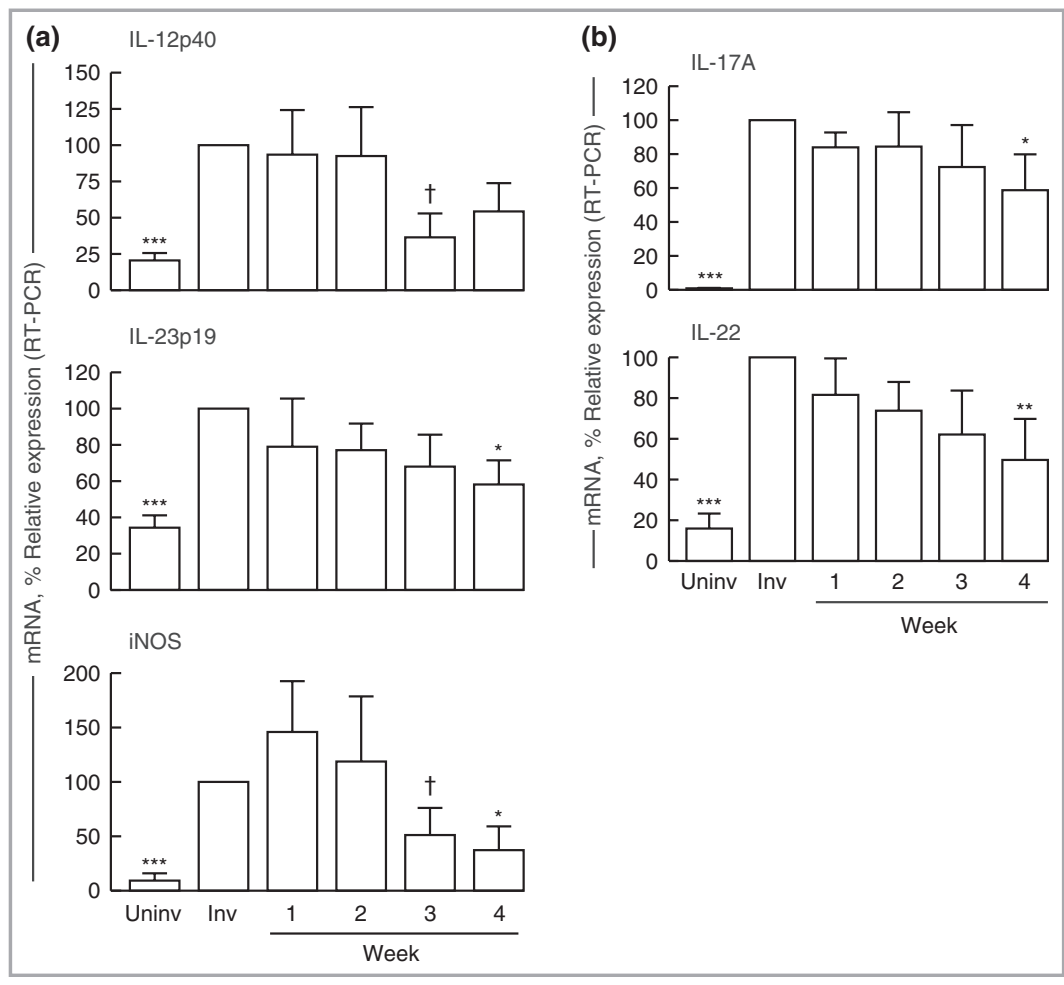

Fig 4. Suppression of inflammatory dendritic cell and Th17 activation by 3-4 weeks of etanercept therapy. (a) In uninvolved (Uninv) and involved (Inv) skin at baseline, as well as involved skin at the indicated weeks of therapy, the effect of etanercept on inflammatory dendritic cells was assessed by using real-time reverse transcriptasepolymerase chain reaction (RT-PCR) to measure expression of interleukin (IL)-12p40 $(\mathrm{n}=20), \mathrm{IL}-23 \mathrm{p} 19(\mathrm{n}=17)$ and inducible nitric oxide synthase (iNOS, $n=16$ ). (b) The effect of etanercept on Th17 cells was assessed by using real-time RT-PCR to measure expression of IL-17A ( $\mathrm{n}=12)$ and IL-22 $(\mathrm{n}=16)$. Data are derived from clinical responders, presented as percentage expression relative to baseline involved skin (normalized to $100 \%$ ), and shown as means \pm SEM. $\dagger \mathrm{P}=0.05, * \mathrm{P}<0.05$, $* * \mathrm{P}<0.01, * * * \mathrm{P}<0.001$, compared with baseline involved skin.
During 4 weeks of treatment, staining of these markers in lesional epidermis and dermis displayed variable changes, decreasing in some subjects while increasing in others. Mean staining did not change significantly for any of these markers (Fig. S4; see Supporting Information).

As lesional $\mathrm{T}$ cells remained stable, we next examined whether their activation was affected by therapy. With 4 weeks of therapy, gene expression of various markers of Tcell function did not change, including Th1 cytokines (interferon- $\gamma$, IL-15), proliferative factors (IL-2), immunosuppressive cytokines (IL-10) and regulatory T-cell function (FOXP3) (data not shown). However, we observed significant changes in cytokine expression of Th17 cells. Indeed, gene expression of IL-17A, which was elevated 113-fold ( $<<0.001$, Table 1) in untreated plaques, did not change with 3 weeks of therapy, but became significantly suppressed by 4 weeks $(P=0 \cdot 03$, Fig. 4b). Similarly, gene expression of IL-22 was elevated in untreated plaques $(P<0.001$, Table 1$)$, but did not decrease significantly until week 4 ( $\mathrm{P}=0 \cdot 004$, Fig. $4 \mathrm{~b})$.

\section{Coordinate effects of tumour necrosis factor- $\alpha$ and interleukin (IL)-17A on induction of the IL-20 subfamily in keratinocytes}

In vivo, we found that expression of IL-19, IL-20 and IL-24 was derived predominantly from psoriatic epidermis (Fig. 2b) and acutely suppressed by etanercept, concomitant with a reduction in epidermal hyperplasia. To gain further insight into the role of TNF- $\alpha$, and therefore etanercept, in psoriasis, we treated NHEKs with TNF- $\alpha$ in the presence or absence of IL-17A. When NHEKs were incubated with IL-17A alone, none of the IL-20 subfamily members was induced. When NHEKs were incubated with TNF- $\alpha$ alone, only IL-20 was induced (Fig. 5). Interestingly, incubation of NHEKs with both TNF- $\alpha$ and IL-17A led to a greater and/or synergistic effect, in which IL-19, IL-20 and IL-24 were induced beyond that seen with TNF- $\alpha$ or IL-17A alone (Fig. 5).

\section{Discussion}

In psoriasis, keratinocyte products stimulate leucocytes and vice versa. ${ }^{1,40}$ Elucidating the pathways that mediate this crosstalk is crucial for gaining insight into disease pathogenesis. To this end, we investigated early biochemical and cellular effects of etanercept on psoriasis lesions prior to substantial clinical improvement. We found that etanercept's earliest target appears to be epidermal keratinocytes.

Our data indicate that etanercept suppresses expression of IL-19, IL-20, IL-24 and numerous keratinocyte markers/products by 1 week of therapy. Subsequently, by $1-4$ weeks, epidermal thickness/hyperplasia improves, translating into flattening of lesions. Numerous studies suggest that the IL-20 subfamily members stimulate epidermal hyperplasia, which is associated with increased expression of CK16 and other psoriasis-associated markers, such as STAT3. ${ }^{15,26}$

As such, we propose that acute suppression of the IL-20 subfamily members and their signalling elements (STAT3) by etanercept reduces epidermal hyperplasia. Normalization of IL-20R1 and IL-20R2 occurs later, suggesting that early improvement of epidermal hyperplasia is mediated by etanercept's targeting of the IL-20 subfamily members, rather than their receptors. Rapid suppression of AREG and HB-EGF, along 


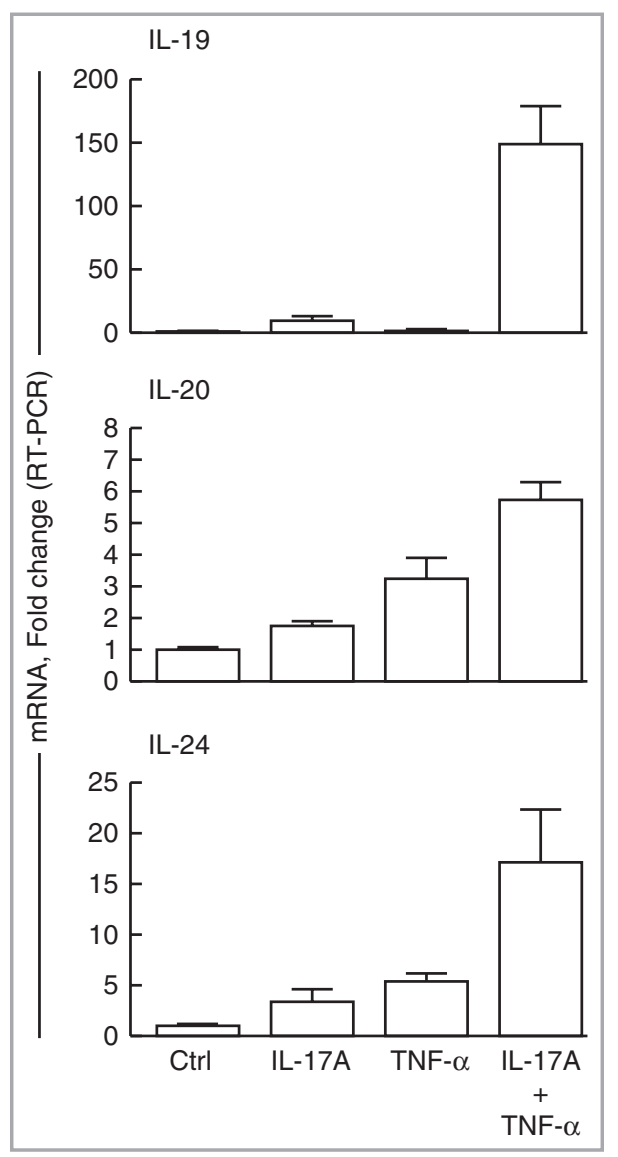

Fig 5. Tumour necrosis factor (TNF)- $\alpha$ and interleukin (IL)-17A coordinately induce the IL-20 subfamily in cultured keratinocytes. Postconfluent normal human epidermal keratinocytes (NHEKs) were incubated for $24 \mathrm{~h}$ with no cytokines (control), IL-17A alone $\left(10 \mathrm{ng} \mathrm{mL} L^{-1}\right)$, TNF- $\alpha$ alone $\left(1 \mathrm{ng} \mathrm{mL} L^{-1}\right)$, or IL-17A $\left(10 \mathrm{ng} \mathrm{mL}^{-1}\right)$ plus TNF- $\alpha\left(1 \mathrm{ng} \mathrm{mL} L^{-1}\right)$. For each condition, six plates of NHEKs were analysed (reflecting two separate experiments, in which each condition was run in triplicate). Real-time reverse transcriptasepolymerase chain reaction (RT-PCR) was then used to assess the expression of IL-19, IL-20 and IL-24. Data are presented as expression relative to control and shown as mean fold change \pm SEM.

with later suppression of IL-22, is likely to contribute to decreased hyperplasia. ${ }^{41,42}$

Sources of IL-19, IL-20 and IL-24 include keratinocytes and leucocytes, with IL-20 produced by monocytes and inflammatory CD $11 \mathrm{c}+\mathrm{DCs}$, and IL-19 and IL-24 produced by activated monocytes. ${ }^{17-19,21-24}$ Using LCM, we found that expression of IL-19, IL-20 and IL-24 was substantially greater in lesional epidermis rather than dermis, where DCs and monocytes/macrophages are found. Thus, acute suppression of the IL-20 subfamily is likely to reflect etanercept's preferential, early targeting of keratinocytes.

Furthermore, rapid epidermal suppression of the IL-20 subfamily is likely to represent a direct consequence of etanercept's antagonism of TNF- $\alpha$. Indeed, consistent with a recent report, ${ }^{43}$ we found that TNF- $\alpha$ combined with IL-17A stimulates synthesis of IL-19, IL-20 and IL-24 in keratinocytes in vitro. This finding indicates that TNF- $\alpha$, in combination with T-cell products, has direct stimulatory effects on psoriatic epidermis, and suggests that antagonism of TNF- $\alpha$ in vivo leads to downregulation of the IL-20 subfamily.

Together, our data indicate that etanercept rapidly suppresses epidermal hyperplasia and activation. Further supporting this concept is our observation that etanercept acutely downregulates other keratinocyte products by 1-2 weeks, including IL-1 $\beta$, AMPs and chemokines. As many of these keratinocyte markers are known to be direct targets of TNF- $\alpha$, their rapid downregulation is likely to reflect antagonism of TNF- $\alpha$ by etanercept. ${ }^{44}$

Following suppression of keratinocyte activation, etanercept appears to target cytokine expression of DCs and $\mathrm{T}$ cells. CD $11 \mathrm{c}+$ inflammatory DCs produce TNF- $\alpha$, iNOS, IL-20 and IL-23, the last of which sustains Th17 polarization. ${ }^{3}$ Previously, Zaba et al. ${ }^{24}$ demonstrated that etanercept reduces IL-20, iNOS and IL-23 production in inflammatory DCs, which remained stable in number with early therapy. Zaba et al. ${ }^{24,29}$ suggested that decreased cytokine production by DCs leads, in turn, to decreased activity of Th17 cells.

Consistent with those observations, we found that early etanercept therapy decreases activation of inflammatory DCs, as reflected by cytokine production. Etanercept's antagonism of TNF- $\alpha$, which is a stimulatory factor for inflammatory DCs, is likely to explain this finding and provides a mechanistic link between TNF- $\alpha$ inhibition and subsequent Th17 downregulation. ${ }^{24,45}$ As CD $11 \mathrm{c}+$ cells did not decrease in number during 4 weeks of therapy, our data also indicate that etanercept does not cause early DC deletion/apoptosis. Moreover, etanercept appears to target inflammatory DCs later than keratinocytes, as the IL-23 subunits p40 and p19 and iNOS were downregulated at 3-4 weeks.

We arrived at similar conclusions regarding $\mathrm{T}$ cells. Consistent with previous findings, ${ }^{24,29}$ we found that etanercept suppresses cytokine expression of Th17 cells, but does not cause deletion/apoptosis. We propose that suppression of DC activation, resulting from etanercept's antagonism of TNF- $\alpha$, leads to dampened Th17 function. As is the case with DCs, Th17 suppression occurs later than that of keratinocytes, with IL-17A and IL-22 decreasing at 4 weeks.

Given our findings, other effector molecules may play a role in regulating proinflammatory products in psoriasis. For instance, studies indicate that IL-17A and/or IL-22 stimulate production of HBD-2 and various chemokines. ${ }^{10,11,46}$ However, we found that downregulation of Th17 cytokines occurred later than that of HBD-2 and chemokines, opening up the possibility that these proinflammatory products might be suppressed by etanercept's targeting of other cytokines, such as TNF- $\alpha$, the IL-20 subfamily or IL- $1 \beta .^{15,18,23,26,47}$

Overall, our study and that of Zaba et al. ${ }^{24}$ are in general agreement, with both finding that 1 week of etanercept treatment suppresses epidermal hyperplasia and activation. Both studies also found that etanercept rapidly suppresses DC- and Th17-related cytokines, although we observed that suppression of these markers tended to be slower. The differences between 
our study and that of Zaba et al. may be related to criteria for selecting treatment responders (PGA vs. histological examination, respectively), statistical methods (two- vs. one-tailed tests, respectively), and normal biological variation.

In conclusion, based on rapid suppression of regenerative hyperplasia and keratinocyte-derived products, including the IL-20 subfamily, we propose that epidermal activation is an early target of etanercept. Decreased epidermal hyperplasia may be a consequence of acute suppression of the IL-20 subfamily, which is likely to result from etanercept's antagonism of TNF$\alpha$. Furthermore, TNF- $\alpha$ can coordinate with IL-17A to regulate the IL-20 subfamily in keratinocytes, and is known to stimulate IL-23-producing DCs. These observations, in conjunction with etanercept's downregulation of T-cell and DC function/activation, support a role for Th17 elements in psoriasis pathogenesis, a concept validated by the efficacy of IL-12/23 inhibitors such as ustekinumab. Additionally, we propose that the IL-20 subfamily is also important to psoriasis pathogenesis, as well as clinical response to etanercept, including flattening of lesions. While anti-IL-20 antibodies have thus far failed in clinical trials (http://www.clinicaltrials.gov, NCT01261767), this failure might be explained by functional redundancy of the IL-20 subfamily. As such, the IL-20 subfamily, as a group, or its receptors remain therapeutic targets for psoriasis.

\section{What's already known about this topic?}

- Psoriasis is a Th17/Th1-mediated skin disease that often responds to antitumour necrosis factor- $\alpha$ therapies, such as etanercept.

- The molecular mechanisms of etanercept are not known.

\section{What does this study add?}

- We analysed the early ( $\leq 4$ week) biochemical and cellular effects of etanercept on psoriasis lesions in vivo.

- A very early (within 1 week) target of etanercept is epidermal hyperplasia/activation.

- Suppression of hyperplasia is likely to result from acute epidermal downregulation of the interleukin (IL)-20 cytokine subfamily (IL-19, IL-20 and IL-24).

\section{Acknowledgments}

We thank the research subjects who participated in this study; Jennifer Bell, CCRP, our clinical research coordinator; and Trivellore Raghunathan and Maria Larkina for assistance with statistical analyses.

\section{References}

1 Lowes MA, Bowcock AM, Krueger JG. Pathogenesis and therapy of psoriasis. Nature 2007; 445:866-73.
2 Clark RA. Skin-resident T cells: the ups and downs of on site immunity. J Invest Dermatol 2010; 130:362-70.

3 Johnson-Huang LM, McNutt NS, Krueger JG et al. Cytokine-producing dendritic cells in the pathogenesis of inflammatory skin diseases. J Clin Immunol 2009; 29:247-56.

4 Zaba LC, Krueger JG, Lowes MA. Resident and 'inflammatory' dendritic cells in human skin. J Invest Dermatol 2009; 129:302-8.

5 Nestle FO, Kaplan DH, Barker J. Psoriasis. N Engl J Med 2009; 361 : 496-509.

6 Ciric B, El-Behi M, Cabrera R et al. IL-23 drives pathogenic IL-17producing CD8+ T cells. J Immunol 2009; 182:5296-305.

7 Duhen T, Geiger R, Jarrossay D et al. Production of interleukin 22 but not interleukin 17 by a subset of human skin-homing memory T cells. Nat Immunol 2009; 10:857-63.

8 Sonnenberg GF, Fouser LA, Artis D. Border patrol: regulation of immunity, inflammation and tissue homeostasis at barrier surfaces by IL-22. Nat Immunol 2011; 12:383-90.

9 Liang SC, Tan XY, Luxenberg DP et al. Interleukin (IL)-22 and IL17 are coexpressed by Th17 cells and cooperatively enhance expression of antimicrobial peptides. J Exp Med 2006; 203: 2271-9.

10 Nograles KE, Zaba LC, Guttman-Yassky E et al. Th17 cytokines interleukin (IL)-17 and IL-22 modulate distinct inflammatory and keratinocyte-response pathways. Br J Dermatol 2008; 159:1092-102.

11 Harper EG, Guo C, Rizzo H et al. Th17 cytokines stimulate CCL20 expression in keratinocytes in vitro and in vivo: implications for psoriasis pathogenesis. J Invest Dermatol 2009; 129:2175-83.

12 Wolk K, Kunz S, Witte E et al. IL-22 increases the innate immunity of tissues. Immunity 2004; 21:241-54.

13 Boniface K, Bernard FX, Garcia M et al. IL-22 inhibits epidermal differentiation and induces proinflammatory gene expression and migration of human keratinocytes. J Immunol 2005; 174:3695-702.

14 Zheng Y, Danilenko DM, Valdez P et al. Interleukin-22, a T(H)17 cytokine, mediates IL-23-induced dermal inflammation and acanthosis. Nature 2007; 445:648-51.

15 Sa SM, Valdez PA, Wu J et al. The effects of IL-20 subfamily cytokines on reconstituted human epidermis suggest potential roles in cutaneous innate defense and pathogenic adaptive immunity in psoriasis. J Immunol 2007; 178:2229-40.

16 Res PC, Piskin G, de Boer OJ et al. Overrepresentation of IL-17A and IL-22 producing CD8 $\mathrm{T}$ cells in lesional skin suggests their involvement in the pathogenesis of psoriasis. PLOS ONE 2010; 5:e14108.

17 Romer J, Hasselager E, Norby PL et al. Epidermal overexpression of interleukin-19 and -20 mRNA in psoriatic skin disappears after short-term treatment with cyclosporine A or calcipotriol. J Invest Dermatol 2003; 121:1306-11.

18 Wang F, Lee E, Lowes MA et al. Prominent production of IL-20 by CD68+/CD11c+ myeloid-derived cells in psoriasis: gene regulation and cellular effects. J Invest Dermatol 2006; 126:1590-9.

19 Kunz S, Wolk K, Witte E et al. Interleukin (IL)-19, IL-20 and IL-24 are produced by and act on keratinocytes and are distinct from classical ILs. Exp Dermatol 2006; 15:991-1004.

20 Tohyama M, Hanakawa Y, Shirakata Y et al. IL-17 and IL-22 mediate IL-20 subfamily cytokine production in cultured keratinocytes via increased IL-22 receptor expression. Eur J Immunol 2009; 39:2779-88.

21 Nagalakshmi ML, Murphy E, McClanahan T et al. Expression patterns of IL-10 ligand and receptor gene families provide leads for biological characterization. Int Immunopharmacol 2004; 4:577-92.

22 Wolk K, Kunz S, Asadullah K et al. Cutting edge: immune cells as sources and targets of the IL-10 family members? J Immunol 2002; 168:5397-402. 
23 Wolk K, Witte K, Witte E et al. Maturing dendritic cells are an important source of IL-29 and IL-20 that may cooperatively increase the innate immunity of keratinocytes. J Leukoc Biol 2008; 83:1181-93.

24 Zaba LC, Cardinale I, Gilleaudeau P et al. Amelioration of epidermal hyperplasia by TNF inhibition is associated with reduced Th17 responses. J Exp Med 2007; 204:3183-94.

25 Parrish-Novak J, Xu W, Brender T et al. Interleukins 19, 20, and 24 signal through two distinct receptor complexes. Differences in receptor-ligand interactions mediate unique biological functions. J Biol Chem 2002; 277:47517-23.

26 Wolk K, Haugen HS, Xu W et al. IL-22 and IL-20 are key mediators of the epidermal alterations in psoriasis while IL-17 and IFNgamma are not. J Mol Med 2009; 87:523-36.

27 Stoler A, Kopan R, Duvic M et al. Use of monospecific antisera and cRNA probes to localize the major changes in keratin expression during normal and abnormal epidermal differentiation. J Cell Biol 1988; 107:427-46.

28 Mansbridge JN, Knapp AM, Strefling AM. Evidence for an alternative pathway of keratinocyte maturation in psoriasis from an antigen found in psoriatic but not normal epidermis. J Invest Dermatol 1984; 83:296-301.

29 Zaba LC, Suárez-Fariñas M, Fuentes-Duculan J et al. Effective treatment of psoriasis with etanercept is linked to suppression of IL-17 signaling, not immediate response TNF genes. J Allergy Clin Immunol 2009; 124:1022-10.

30 Kang S, Cho S, Chung JH et al. Inflammation and extracellular matrix degradation mediated by activated transcription factors nuclear factor-kappaB and activator protein-1 in inflammatory acne lesions in vivo. Am J Pathol 2005; 166:1691-9.

31 Quan T, Qin Z, Xia W et al. Matrix-degrading metalloproteinases in photoaging. J Investig Dermatol Symp Proc 2009; 14:20-4.

32 Elder JT, Fisher GJ, Zhang QY et al. Retinoic acid receptor gene expression in human skin. J Invest Dermatol 1991; 96:425-33.

33 Moore A, Gordon KB, Kang S et al. A randomized, open-label trial of continuous versus interrupted etanercept therapy in the treatment of psoriasis. J Am Acad Dermatol 2007; 56:598-603.

34 Langley RG, Ellis CN. Evaluating psoriasis with Psoriasis Area and Severity Index, Psoriasis Global Assessment, and Lattice System Physician's Global Assessment. J Am Acad Dermatol 2004; 51:563-9.

35 Otkjaer K, Kragballe K, Funding AT et al. The dynamics of gene expression of interleukin-19 and interleukin-20 and their receptors in psoriasis. Br J Dermatol 2005; 153:911-18.

36 Blumberg H, Conklin D, Xu WF et al. Interleukin 20: discovery, receptor identification, and role in epidermal function. Cell 2001; 104:9-19.

37 Dumoutier L, Leemans C, Lejeune D et al. Cutting edge: STAT activation by IL-19, IL-20 and mda-7 through IL-20 receptor complexes of two types. J Immunol 2001; 167:3545-9.

38 Baker BS, Swain AF, Fry L et al. Epidermal T lymphocytes and HLADR expression in psoriasis. Br J Dermatol 1984; 110:555-64.

39 Bos JD, Hulsebosch HJ, Krieg SR et al. Immunocompetent cells in psoriasis. In situ immunophenotyping by monoclonal antibodies. Arch Dermatol Res 1983; 275:181-9.

40 Krueger JG. The immunologic basis for the treatment of psoriasis with new biologic agents. J Am Acad Dermatol 2002; 46:1-23.

41 Bhagavathula N, Nerusu KC, Fisher GJ et al. Amphiregulin and epidermal hyperplasia: amphiregulin is required to maintain the psoriatic phenotype of human skin grafts on severe combined immunodeficient mice. Am J Pathol 2005; 166:1009-16.

42 Piepkorn M, Predd H, Underwood R et al. Proliferation-differentiation relationships in the expression of heparin-binding epidermal growth factor-related factors and erbB receptors by normal and psoriatic human keratinocytes. Arch Dermatol Res 2003; 295:93101.

43 Chiricozzi A, Guttman-Yassky E, Suárez-Fariñas M et al. Integrative responses to IL-17 and TNF- $\alpha$ in human keratinocytes account for key inflammatory pathogenic circuits in psoriasis. J Invest Dermatol $2011 ; 131: 677-87$.

44 Haider AS, Cohen J, Fei J et al. Insights into gene modulation by therapeutic TNF and IFNgamma antibodies: TNF regulates IFNgamma production by $\mathrm{T}$ cells and TNF-regulated genes linked to psoriasis transcriptome. J Invest Dermatol 2008; 128:655-66.

45 Lee E, Trepicchio WL, Oestreicher JL et al. Increased expression of interleukin 23 p19 and p40 in lesional skin of patients with psoriasis vulgaris. J Exp Med 2004; 199:125-30.

46 Guilloteau K, Paris I, Pedretti N et al. Skin inflammation induced by the synergistic action of IL-17A, IL-22, oncostatin M, IL- $1 \alpha$, and TNF- $\alpha$ recapitulates some features of psoriasis. J Immunol 2010; 184:5263-70.

47 Schroder JM, Harder J. Human beta-defensin-2. Int J Biochem Cell Biol 1999; 31:645-51.

\section{Supporting Information}

Additional Supporting Information may be found in the online version of this article:

Fig S1. Normalization of several receptor subunits for the interleukin (IL)-20 subfamily during the first month of etanercept therapy. In uninvolved (Uninv) and involved (Inv) skin at baseline, as well as involved skin at the indicated weeks of therapy, real-time reverse transcriptase-polymerase chain reaction (RT-PCR) was used to assess the expression of receptor subunits for the IL-20 subfamily of cytokines, including IL20R1, IL-20R2 and IL-22R1 (all $n=8$ ). Data are derived from clinical responders, presented as percentage expression relative to baseline involved skin (normalized to $100 \%$ ), and shown as means \pm SEM. $* \mathrm{P}<0.05, * * * \mathrm{P}<0.001$, compared with baseline involved skin.

Fig S2. Rapid suppression of keratinocyte activation by 12 weeks of etanercept therapy. In uninvolved (Uninv) and involved (Inv) skin at baseline, as well as involved skin at the indicated weeks of therapy, the effect of etanercept on keratinocyte activation/function was assessed by using real-time reverse transcriptase-polymerase chain reaction (RT-PCR) to measure expression of $(\mathrm{a}-\mathrm{d})$ the chemokines CXCL1 $(\mathrm{n}=11)$, CXCL8 $(n=16)$, CCL20 $(n=12)$ and CXCL10 $(n=11) ;(e)$ the proinflammatory cytokine interleukin (IL)- $1 \beta \quad(n=16)$; $(\mathrm{f}-\mathrm{h})$ the antimicrobial peptides human cathelicidin antimicrobial peptide-18/eucine-leucine-37 (hCAP-18/LL-37) $(\mathrm{n}=11)$, human $\beta$-defensin (HBD)-2 ( $\mathrm{n}=10)$ and HBD-3 $(\mathrm{n}=7)$; and $(\mathrm{i}-\mathrm{j})$ the keratinocyte growth factors amphiregulin (AREG, $\mathrm{n}=16$ ) and heparin-binding epidermal growth factor-like growth factor (HB-EGF, $\mathrm{n}=16$ ). Data are derived from clinical responders, presented as percentage expression relative to baseline involved skin (normalized to $100 \%$ ), and shown as means \pm SEM. $* \mathrm{P}<0.05, * * \mathrm{P}<0.01, * * * \mathrm{P}<0.001$, compared with baseline involved skin.

Fig S3. Inflammatory dendritic cells do not decrease significantly during the first month of etanercept therapy. In uninvolved (Uninv) and involved (Inv) skin at baseline, as well as 
102 Etanercept acutely suppresses the IL-20 cytokine subfamily, F. Wang et al.

involved skin at the indicated weeks of therapy, area of dermal immunostaining of $\mathrm{CD} 11 \mathrm{c}(\mathrm{n}=7)$ was quantified. Data are derived from clinical responders, and are presented as percentage dermal area stained. $* \mathrm{P}<0.01$.

Fig S4. Lesional T-cell numbers remain stable during the first month of etanercept therapy. In uninvolved (Uninv) and involved (Inv) skin at baseline, as well as involved skin at the indicated weeks of therapy, area of epidermal and dermal immunostaining of $\mathrm{CD} 3, \mathrm{CD} 4$ and $\mathrm{CD} 8$ (all $\mathrm{n}=7$ ) was quanti- fied. Data are derived from clinical responders, presented as percentage area stained relative to baseline involved skin (normalized to $100 \%$ ), and shown as means \pm SEM. $* \mathrm{P}<0.05$, $* * \mathrm{P}<0.01, * * * \mathrm{P}<0.001$, compared with baseline involved skin.

Please note: Wiley-Blackwell are not responsible for the content or functionality of any supporting materials supplied by the authors. Any queries (other than missing material) should be directed to the corresponding author for the article. 